\title{
Amplitude and intensity interferometry using satellite LNB receivers for innovative and low cost microwave and millimetre wave sensor development
}

\author{
Neil A. Salmon*, Joel Radiven, Peter N. Wilkinson \\ Jodrell Bank Centre for Astrophysics, University of Manchester, Manchester, M13 9PL, UK
}

\begin{abstract}
Satellite Low Noise Block-down convertors (LNBs) have been evaluated for use in amplitude and intensity interferometry. LNBs have been found to have a high performance to cost ratio which is beneficial for any sensor system. They are investigated here for a diversity of applications from the derisking of subsystems for next generation aperture synthesis imagers having hundreds of channels ${ }^{[1]}$ to a platform for the investigation of phase recovery in intensity interferometry and experimentation in entangled photons. Measured noise temperatures of LNBs were found to lie between $170 \mathrm{~K}$ and $300 \mathrm{~K}$ which is higher than typical manufacturers' specifications. A twin channel interferometer system was developed using satellite receiver feeds and LNBs at the front-end, other amplifiers, mixers, filters and local oscillators at intermediate stages, and 8-bit USB ADCs sampling synchronously at $100 \mathrm{MHz}$ and a $\mathrm{PC}$ for data processing. LabVIEW was used to digitally demodulate the sampled data and process it into the first and second orders of coherence. Measurements of the first order of coherence from a standard low energy discharge lamp indicated interference fringes were commensurate with range and spacing of the two receivers and the source. The relationship between the measured first and second order of coherence agrees within the experimental error. Variations of the first and second orders of coherence with range, $R$, follow the relationship $1 / R$ and $1 / R^{2}$. The system has the potential for investigations into phase extraction for intensity interferometry and for the study of digital demodulation schemes for aperture synthesis amplitude interferometry with hundreds of receiver channels for next generation security screening systems. A twin, triple or quadruple channel polarimetric LNB interferometer could be used as basis for high precision investigations in to entangled photons and quantum communications.
\end{abstract}

Keywords: Aperture synthesis, imaging, coherence, security screening, digital, passive, satellite, LNB

\section{INTRODUCTION}

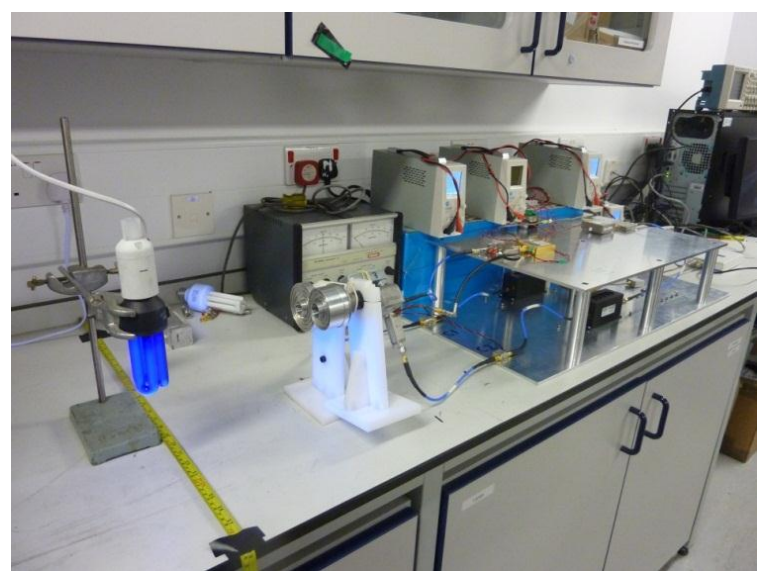

Figure 1: The twin channel $12 \mathrm{GHz}$ LNB combined amplitude and intensity interferometry system

A number of aperture synthesis imaging systems have been developed to derisk the development of sensors with a large number (300) of receivers with the aim of producing imagery with 20,000 pixels sufficient for security screening of personnel ${ }^{[1]}$. This particular project investigates whether the low cost $(£ 10$ each) LNB satellite receivers have sufficient capability for such a future sensor. Furthermore, in this project schemes for band-pass $f_{s} / 4$ sampling and digital downconversion can be investigated using LabVIEW as a route to derisking these schemes which can later be implemented in hardware to achieve much higher bandwidths and sampling frequencies in future imagers. By way of system evaluation the first and second degrees of coherence were evaluated. The system development was also motivated by the interest in whether phase information can be extracted from the second order of coherence of intensity interferometry. The authors are also aware that this architecture might prove useful in the search for a source of entangled photons that might be used in quantum communication systems. 


\subsection{Components}

\section{SYSTEM DESCRIPTION}

The interferometer, shown in Figure 1, comprises two stages of heterodyne downshifting, the first taking place in the satellite LNBs (each costing £10 each) and the second taking place at a lower frequency, as detailed in the block diagram of Figure 2. The LNB provides front end amplification over the band 10 to $12 \mathrm{GHz}$ followed by the down mixing, for which a $10 \mathrm{GHz}$ external local oscillator was injected, and finally intermediate frequency (IF) amplification

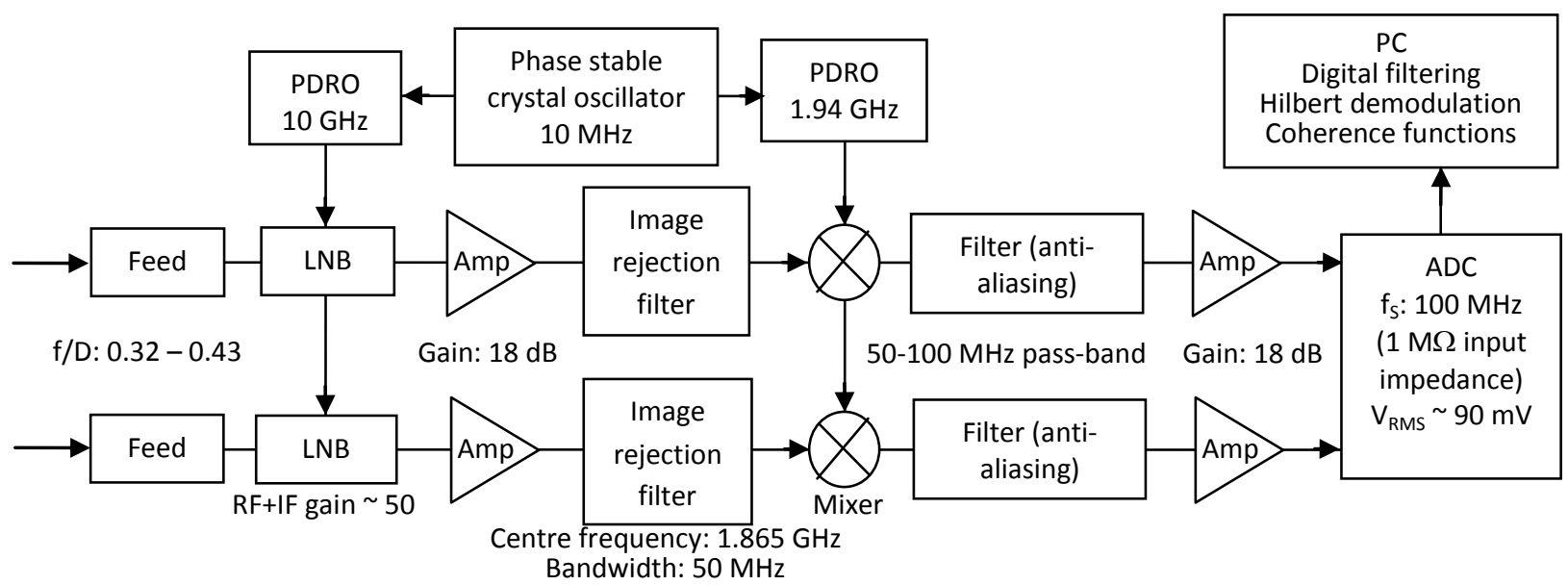

Figure 2: The block diagram of the twin channel $12 \mathrm{GHz}$ LNB combined amplitude and intensity interferometer

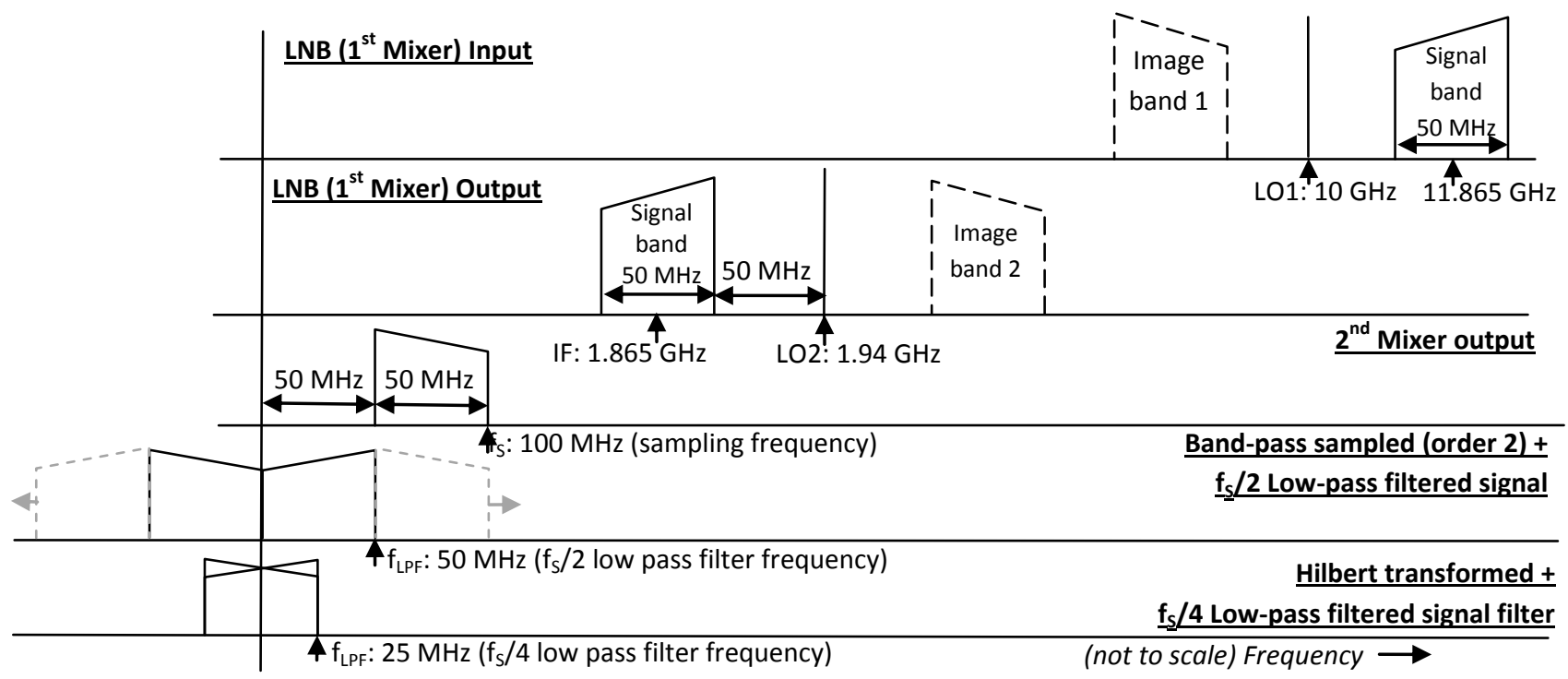

Figure 3: The spectral signal content in the analogue and digital down-shifting processes of the interferometer

over the band from a few $100 \mathrm{MHz}$ to just over two GHz. The LNB has a total gain measured in the region of $50 \mathrm{~dB}$ and to boost the signal in the IF band it was followed by a low cost (£2.50) amplifier with a gain of $\sim 18 \mathrm{~dB}$. The second stage mixers use a local oscillator at $1.94 \mathrm{GHz}$, using a pass-band filter centred at $1.865 \mathrm{GHz}$ to reject the image band from the band $1.99 \mathrm{GHz}$ to $2.04 \mathrm{GHz}$. Signals output from the second mixer are pass band filtered from a band $50 \mathrm{MHz}$ to $100 \mathrm{MHz}$ prior to bandpass sampling (of order $p=2$ ) at a sample frequency of $100 \mathrm{MHz}{ }^{[2]}$. The down-shifting processes in this sensor scheme are illustrated in Figure 3. Together with the filtering, the system is sensitive in the radio frequency $(\mathrm{RF})$ section at a centre frequency of $11.865 \mathrm{GHz}$ with a bandwidth of $50 \mathrm{MHz}$. 
The radio frequency bandwidths of universal satellite LNBs extends from $10.7 \mathrm{GHz}$ to $12.75 \mathrm{GHz}$, this being accessible using two switchable local oscillators internal to the LNB at frequencies of $9.75 \mathrm{GHz}$ and $10.60 \mathrm{GHz}($ set by a $0 / 22 \mathrm{KHz}$ tone), these putting the IF bands at $0.95 \mathrm{GHz}$ to $1.95 \mathrm{GHz}$ and $1.1 \mathrm{GHz}$ to $2.15 \mathrm{GHz}$ respectively ${ }^{[3]}$. For the system to operate as an amplitude interferometer, these internal oscillators must be disconnected, and a common external local oscillator injected into each LNB. This requires careful work at a static free work station in which the internal oscillator track on the LNB circuit is broken and the core and screen of a coaxial line from the external oscillator soldered in its place, as illustrated in Figure 4.

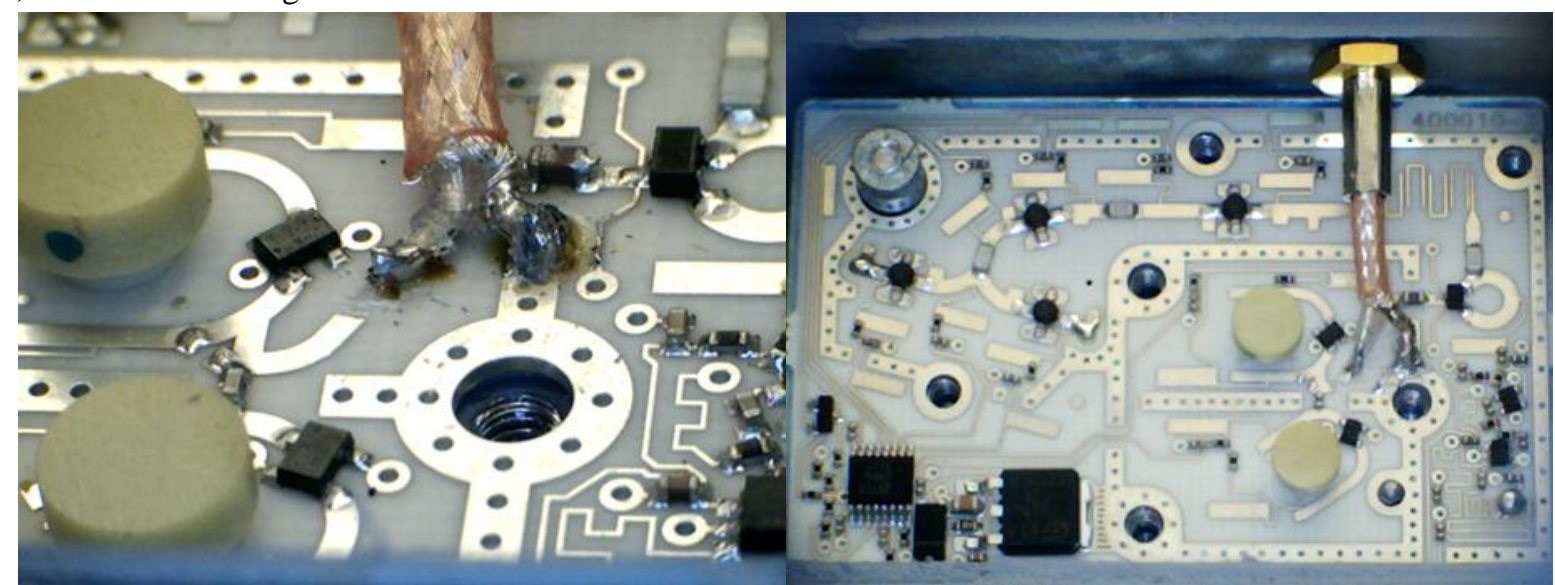

Figure 4: Disconnection of the LNB internal local oscillators and injection of the external $10 \mathrm{GHz}$ oscillator

A further requirement for interferometry is for the sampling of the data on the two channels to be synchronous, and this is achieved using a National Instrument USB 5133 ADC. It samples on two channels each at 8-bits and at $100 \mathrm{MHz}$, and has $32 \mathrm{MB}$ of storage.

\subsection{Digital down conversion and the Hilbert transform}

The effect of sampling the signal creates multiple replicas of the signal in frequency space and the lowest of these is digitally filtered out using a low pass filter with a cut-off frequency of $50 \mathrm{MHz}$. Effectively this process has downshifted the analogue signal by $f_{S} / 2$. As the correlation function is by definition complex, an imaginary component to the digitised signal now has to be created. This is done by performing the Hilbert transform (Eq. 1) on the sampled data $r(t)$ which is then used to build the analytic signal, $A(t)$, as in Eq. 2. All signal information is now contained in the band from DC to $25 \mathrm{MHz}$, the Hilbert transform effectively having down-shifted the signal by $f_{S} / 4$. This signal is then digitally low pass filtered with a cut-off frequency of $25 \mathrm{MHz}^{[4]}$. Processing on the digitised signals all takes place in LabVIEW with the spectra of down-shifted signal being shown in Figure 3.

$$
H(t)=\frac{1}{\pi} \int_{-\infty}^{+\infty} \frac{r(\tau)}{(t-\tau)} d \tau \quad(1) \quad A(t)=r(t)-j H(t)
$$

\subsection{Coherence length}

\section{COHERENCE FUNCTIONS}

The temporal coherence length of natural passive radiometric emission is very short, typically on the scale of the radiative transition timescales which are at a fraction of a nano-second, this being a wave train in space a few centimetres long ${ }^{[7]}$. However, once natural emission has been filtered, the narrowing of the radiation bandwidth, $\mathrm{B}_{\mathrm{RF}}$ imposes an artificial coherence length on the radiation set by Eq. 3. For the system described here, this coherence length is of the order of $6 \mathrm{~m}$. Key parameters in the characterisation of sources and in interferometric imaging are the orders of coherence.

$$
l_{C}=\frac{c}{B_{R F}}
$$




\subsection{Degree of first order coherence (amplitude interferometry)}

In amplitude interferometry the key parameter generated by the sensor is the degree of first order coherence, which is the mean value of the product of electric field $E_{l}$ measured at one antenna location with the conjugate of the field $E_{2}$ at the other antenna location ${ }^{[8]}$. It is a complex quantity, defined mathematically in Eq. 4, where $\tau$ is a lag time between the two sampled signals, which in this system is zero and the angle brackets indicate the long term mean value, which is calculated over an integration period of $30 \mathrm{~ms}$, during which $3 \mathrm{M}$ data points are sampled. This mean value is normalised by dividing by the root mean square of each of the electric fields in the two channels, these values being calculated using LabVIEW functions, the circuit block diagrams for this being shown in Figure 5. For two identical signals the degree of first order coherence is unity, whereas for completely uncorrelated signals the coherence is zero.

$$
\gamma_{1,2}^{(1)}(\tau)=\frac{\left\langle E_{1}(t+\tau) E_{2}^{*}(t)\right\rangle}{\sqrt{\left\langle\left|E_{1}(0)\right|^{2}\right\rangle\left\langle\left|E_{2}(0)\right|^{2}\right\rangle}}
$$

The first order of coherence is that function used in standard radio astronomy aperture synthesis, where it is used to build up the visibility function by placing these samples on a two dimensional grid of base-lines (antenna pair separations / divided by the radiation wavelength) ${ }^{[6]}$. Taking the Fourier transform of the visibility function gives the image of the scene. For this system however, the variation of $\gamma^{(1)}$ will be investigated by varying source locations, to evaluate the effectiveness of this scheme of interferometric sensor development.

\subsection{Degree of second order coherence (intensity interferometry)}

In intensity interferometry the key parameter is the degree of second order coherence, which is the normalised mean value of the product of the two intensities $I_{1}$ and $I_{2}$ measured at the two antenna locations ${ }^{[9]}$. It is a purely real quantity, defined mathematically by Eq. 5 where $I(t)$ is the short term (cycled average) intensity, and as above the time lag $\tau$ is zero. Normalisation is achieved through division by the long term time averaged intensities in the separate channels and these functions are all calculated using the mathematical functions in LabVIEW, the circuit block diagram for these calculations being shown in Figure 6. As above with amplitude interferometry $30 \mathrm{~ms}$ of data is recorded, a total of $3 \mathrm{M}$ sample points. For perfectly correlated short term intensity fluctuations the degree of second order coherence has a value of two, whilst for completely uncorrelated intensities it has a value of unity.

$$
\gamma_{1,2}^{(2)}(\tau)=\frac{\left\langle I_{1}(t+\tau) I_{2}(t)\right\rangle}{\left\langle I_{1}(0)\right\rangle\left\langle I_{2}(0)\right\rangle}
$$

$$
I(t)=E(t) E^{*}(t)
$$

The second order of coherence is that function measured in the Hanbury-Brown and Twiss type experiments of intensity interferometry ${ }^{[5]}$. As the intensity represents a squaring of the amplitude, direct phase information about the incoming wave is lost. However, in the process of squaring the summation of a spatial distribution of electric amplitudes across the source, phase information about the source is retained in cross-terms. Hanbury-Brown and Twiss measured how the second order coherence function varied with delay to estimate stellar diameters. Used in the visible spectrum it proved easier to realise than the Michelson stellar interferometry, as it was less sensitive to atmospheric scintillations. Since then a small community has tried to extract phase information from the intensity interferometer, using the triple product (bispectral) technique ${ }^{[10]}$ and the Cauchy-Riemann phase recovery technique ${ }^{[11]}$, as a means of generating images. If the phase problem is solved his may enable the building of ground based high resolution optical interferometric telescopes that are insensitive to atmospheric scintillations ${ }^{[12]}$. The relationship between the degree of first and second order coherence is given by Eq.7, the phase information from the first order coherence is lost through the squaring process.

$$
\gamma_{1,2}^{(2)}=1+\left|\gamma_{1,2}^{(1)}\right|^{2}
$$




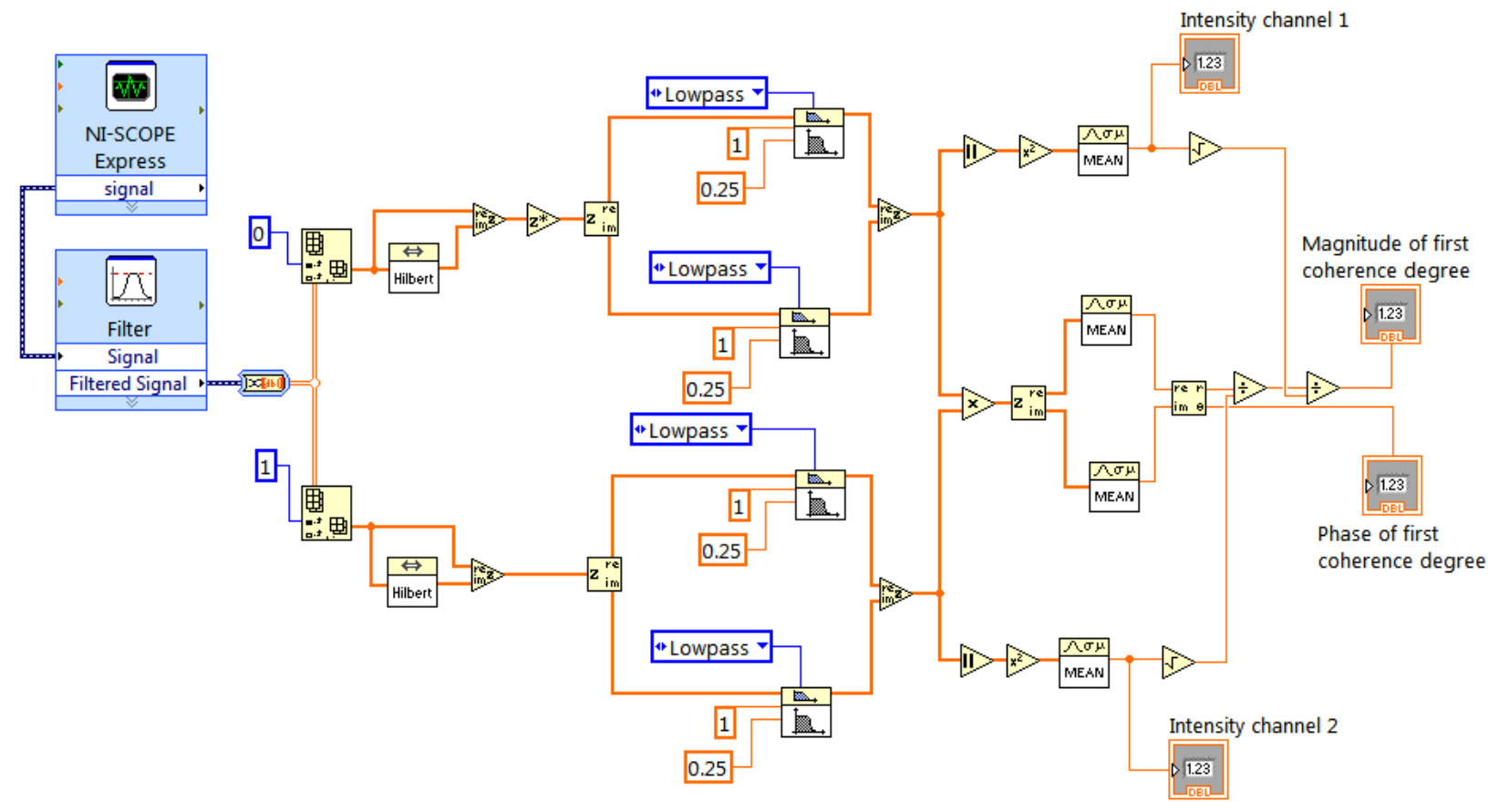

Figure 5: The LabVIEW circuit block diagram for calculation of the degree of first order coherence

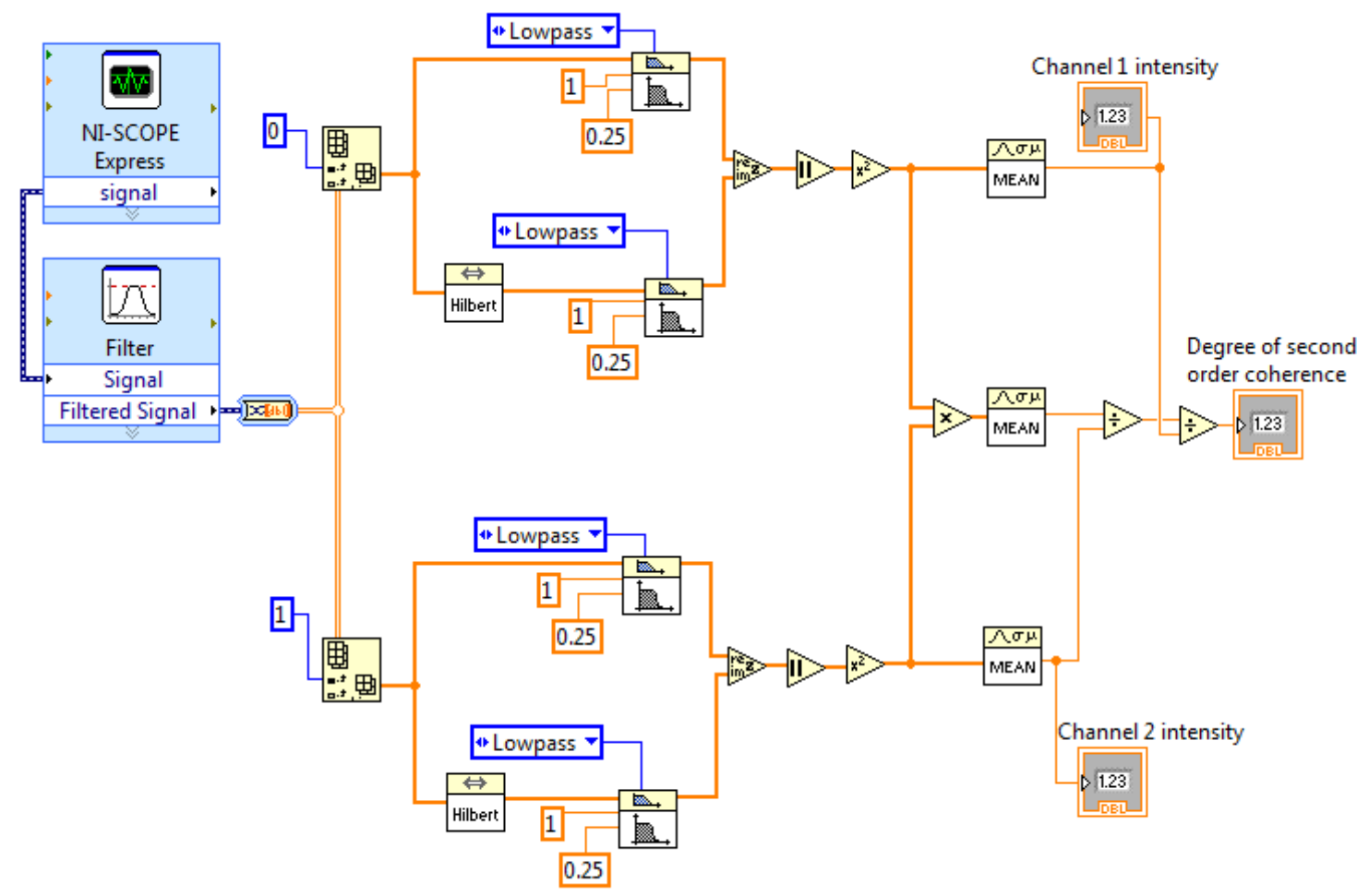

Figure 6: The LabVIEW circuit block diagram for calculation of the degree of second order coherence. 


\subsection{Signal to noise ratios in amplitude and intensity interferometry}

The noise level on the degree of first and second order coherence is given by Eq. 8 and 9, where $B_{C O R R}$ is the correlator bandwidth and $t_{I N T}$ is the integration time ${ }^{[5]}$. The correlator bandwidth is stated as this expression comes from work in optical intensity interferometers. It can be seen from this that the higher the correlator bandwidth the lower the noise. In both cases the time bandwidth product is exactly the number of measurement points. In the case of the intensity interferometer noise becomes smaller with increased correlator bandwidth. In the microwave region the bandwidth of the correlator approaches the RF bandwidth, in which case both the amplitude and intensity interferometers have the same noise levels.

$$
\Delta \gamma_{1,2}^{(1)}=\left(B_{R F} t_{I N T}\right)^{-1 / 2}
$$

$$
\Delta \gamma_{1,2}^{(2)}=\left(B_{C O R R} t_{I N T}\right)^{-1 / 2}
$$

\subsection{Performance of LNBs}

\section{RESULTS}

The noise temperature of the LNB at $11.865 \mathrm{GHz}$ was determined using the y-factor test and a power meter to measure the signal power output from the LNBs when the system was viewing alternately liquid nitrogen and ambient temperature absorber. For a small number of LNBs the receiver noise temperatures were measured at between $170 \mathrm{~K}$ and $300 \mathrm{~K}$. Manufacturers typically quote the noise figures at between $0.1 \mathrm{~dB}$ and $0.3 \mathrm{~dB}$, which are equivalent to noise temperatures of $7.5 \mathrm{~K}$ and $23.5 \mathrm{~K}$. The disagreement between these and the measured figures may be due to the fact that the manufacturers' specifications might be measured at different frequencies and under different circumstances. Using the LNB with the externally injected local oscillator it was possible to measure how the noise temperature of the device varied with local oscillator power, and this is shown in Figure 7. Following this measurement, the power injected to the LNBs for operation was chosen around $10 \mathrm{dBm}$ to give the best noise performance. The spectrum of the output power from the LNB is shown in Figure 7. The peak of the power output is around $1.31 \mathrm{GHz}$, which given the $10 \mathrm{GHz}$ local oscillator frequency corresponds to an RF input of $11.31 \mathrm{GHz}$. There is a strong roll off in response at frequencies above $1.9 \mathrm{GHz}$.
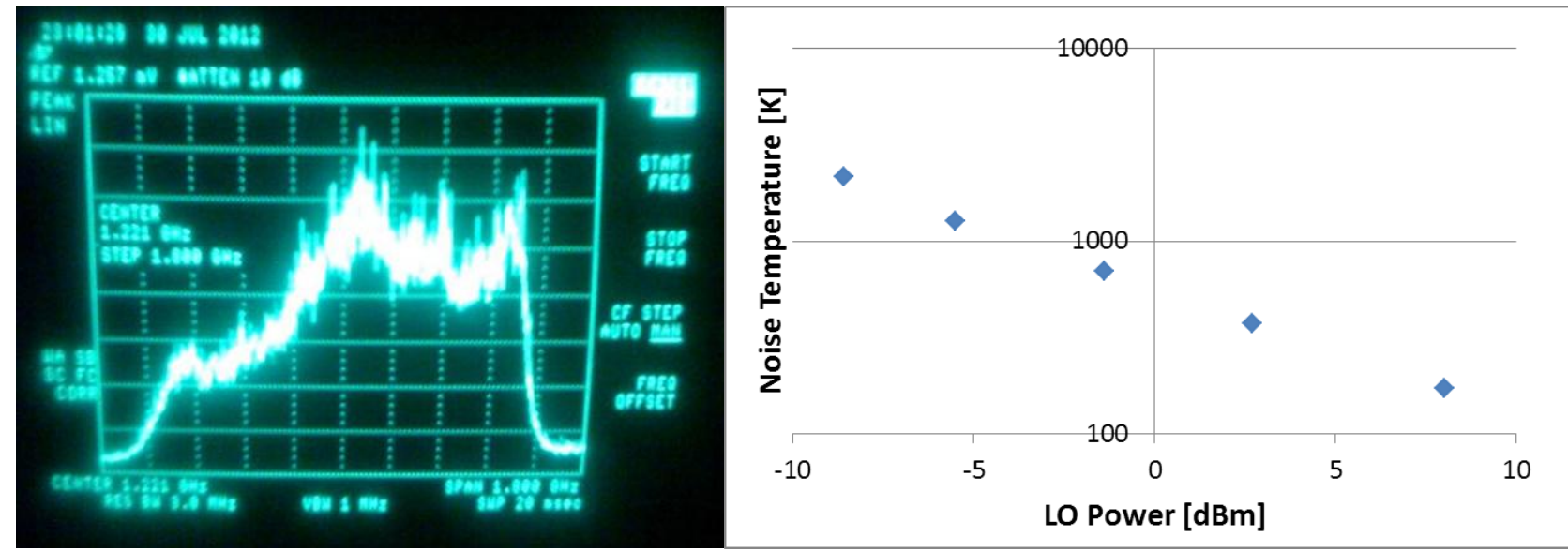

Figure 7: The spectrum of intermediate frequency output power (LHS) from a satellite LNB (centre frequency: 1.22 $\mathrm{GHz}$; span: $1.8 \mathrm{GHz}$ ), the roll off in the response at the higher frequency could be due to the spectrum analyser, which had a maximum operating frequency of $2 \mathrm{GHz}$. The noise temperature of the LNB measured as a function of local oscillator power at an IF of $1.865 \mathrm{GHz}$ (RHS).

\subsection{Performance of $2^{\text {nd }}$ mixer and analogue IF stages}

Validation that analogue components following the LNBs were working properly, a power meter was used to measure signal levels from the liquid nitrogen and ambient temperature absorber sources. Signal levels were in agreement with those expected on the basis of gains and bandwidths in the system. The typical measured power levels $(\sim-9 \mathrm{dBm})$ and rms voltages into the ADCs $(80 \mathrm{mV})$ were consistent with a total gain $G$ of $86 \mathrm{~dB}$, a radiation bandwidth of $50 \mathrm{MHz}$, an antenna temperature $T_{A}$ (taken to be that of the ambient room temperature $\sim 290 \mathrm{~K}$ ), a receiver noise temperature $T_{N}$ (measured to be $170 \mathrm{~K}$ ) and a transmission line impedance $R$ of $50 \mathrm{Ohms}$, as given by Eq. 10 . 


$$
\frac{V_{R M S}^{2}}{R}=k\left(T_{A}+T_{N}\right) B_{R F} G
$$

\subsection{Validation of ADCs and synchronous sampling}

Critical for the interferometer is the validation that the two receiver channels are sampling synchronously, as without this no interference effects will result, as the samples will be outside the coherence length. This validation was performed by examining the response of the system to correlated noise. This measurement was achieved by splitting a single channel of the interferometer into two and inputting these into the two channels of the ADC. It was a simple matter in LabVIEW to visibly check the sampled data side by side. Changes in the signal levels were noted to be the same for each sample from the start of the sample train through to the end of the $3 \mathrm{M}$ sample points. There was however noted a small calibration error on the absolute value recoded on each channel, of the order of $\sim 20 \%$. This was consistent over a period of time, and as such should be calibrated in a future element of the work.

Further assessment of correlated noise was performed by calculating the first and second orders of coherence, these were found to be consistently 0.8 and 1.71 respectively. It is expected from the definitions of the coherence that the first and second coherence orders should be 1.0 and 2.0 respectively for perfectly correlated noise. It is believed these differences are due to the calibration errors on the ADCs.

Also critical for interferometric operation is the validation that uncorrelated noise is indeed measured to be uncorrelated. Examination of this verifies that measured correlations are not the result of cross-talk. For the generation of uncorrelated noise the feed of each LNB was pointed towards separate sections of absorber. The measurements showed the first and second orders of coherence were consistently 0.0009 and 1.0007 , whereas the expectation values are 0.0 and 1.0 respectively. The discrepancy is consistent with fluctuation noise associated with a finite number of measurements, which at a value of reciprocal root $3 \mathrm{M}, \sim 6 \times 10^{-4}$, is close to the measured difference between measurement and expectation.

\subsection{Measurements of the coherence functions}

To validate the system was working as an interferometer the degree of first order and second order coherence functions were measured using emission from a low energy Compact Fluorescent Light (CFL) bulb, whilst the light was being moved around before the feeds.

The phase of the first order of coherence $\gamma^{(1)}$ was measured as the CFL bulb at a range of $13.5 \mathrm{~cm}$ was displaced transversely before the LNB feeds, separated by distance $b$ of $6 \mathrm{~cm}$, as illustrated in Figure 8 . This movement should generate fringes directly in front of the LNB having a spacing given by the radiation wavelength $(2.527 \mathrm{~cm})$ divided by the feed separation, multiplied by the range $R$, which is $\sim 7.84 \mathrm{~cm}$. The phase of the coherence function from Figure 8 indicates the fringe separation is $8.0 \mathrm{~cm}$, in good agreement with the prediction. Greater off-axis angles would be expected to show an increase in fringe spacing and this is shown to be the case from the measurements in the figure. It may also be noted that these measurements are in the near field, as the Rayleigh distance, $2 b^{2} / \lambda$, is $\sim 28 \mathrm{~cm}$.

The Magnitude of the first order coherence function $\gamma^{(1)}$ has been measured at around 0.06, (which is well above the noise level), but there appeared little structure in the variation of this measurement over the displacement before the LNB feeds. Assuming the only emission in the scene is a disk of diameter $D$, at a range $R$, viewed with LNBs spaced by $b$, the first order coherence function is given ${ }^{[8]}$ by Eq. 11, where $J_{l}$ is a Bessel function of the first kind of order one and $\psi$ is the phase term. Substituting the values of $D, R$ and $b$ into this equation gives the magnitude of $\gamma^{(1)}$ at $\sim 0.6$. This is ten times higher than the above measured value. This is possibly due to the fact that the CFL bulb has a complex structure comprising a discharge tube folded in to six sections each having a diameter of just less than a centimetre and emission from the ambient temperature background will cause decorrelating effects.

$$
\gamma_{1,2}^{(1)}=\frac{2}{\pi} \frac{\lambda R}{b D} J_{1}\left(\pi \frac{b D}{\lambda R}\right) \exp i \Psi
$$


The magnitudes of the first and second order coherence, $\gamma^{(1)}$ and $\gamma^{(2)}$, were measured as the CFL bulb was varied in range from the LNBs outwards to a distance of $50 \mathrm{~cm}$, as illustrated in Figure 9. The measurements of the magnitudes of $\gamma^{(1)}$ and $\left(\gamma^{(2)}-1\right)$ are shown as a function of range in Figure 9. The magnitude of, $\gamma^{(1)}$ is greater than $\left(\gamma^{(2)}-1\right)$ by approximately an order of magnitude, which is roughly in agreement with Eq. 7. The lamp has a physical width of the order of $3.5 \mathrm{~cm}$ and variation of the lamp out around $10 \mathrm{~cm}$ showed a dip in coherence. However, from around $10 \mathrm{~cm}$ out to $50 \mathrm{~cm}$ the variation in magnitudes of $\gamma^{(1)}$ and $\left(\gamma^{(2)}-1\right)$ follow approximately a $1 / R$ and a $1 / R^{2}$ relationship, these fits also being shown in Figure 9. Little coherent structure was found in the variation of the measured phase of the first order coherence with range.

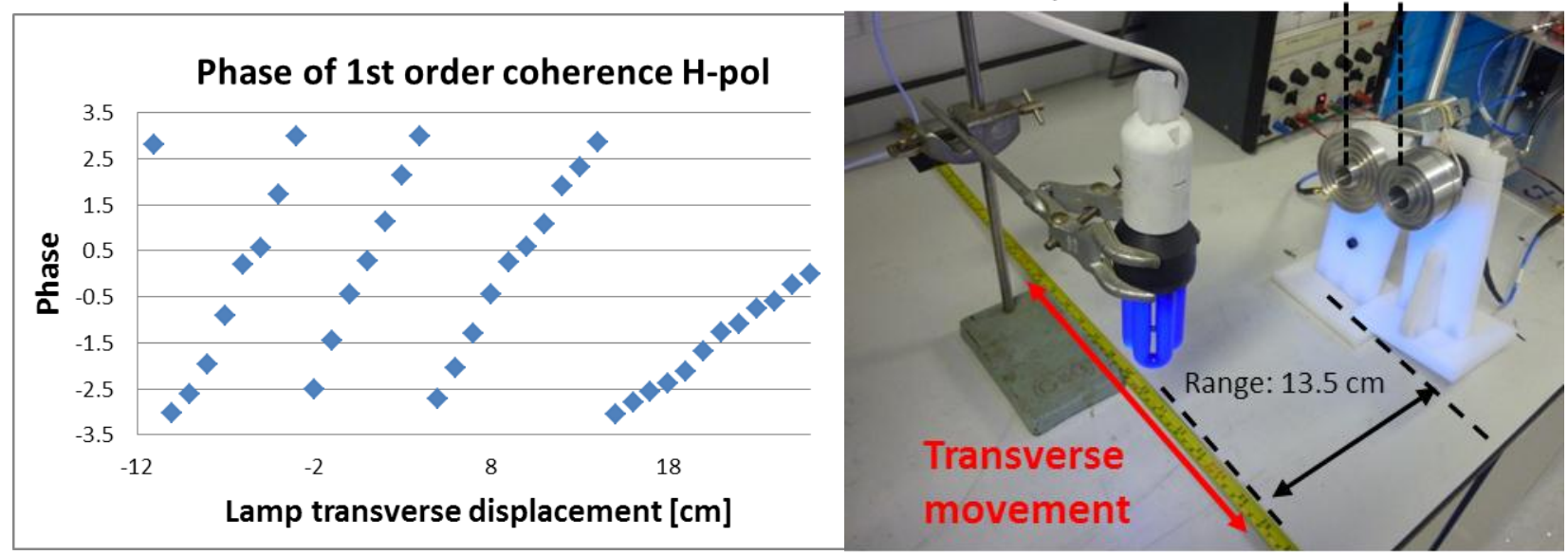

Figure 8: Interference fringes in the phase of the first order coherence function $\gamma^{(1)}$ were detected by measuring the emission from a low energy light bulb as it was displaced before the LNB feeds. Position zero on the $\mathrm{x}$-axis

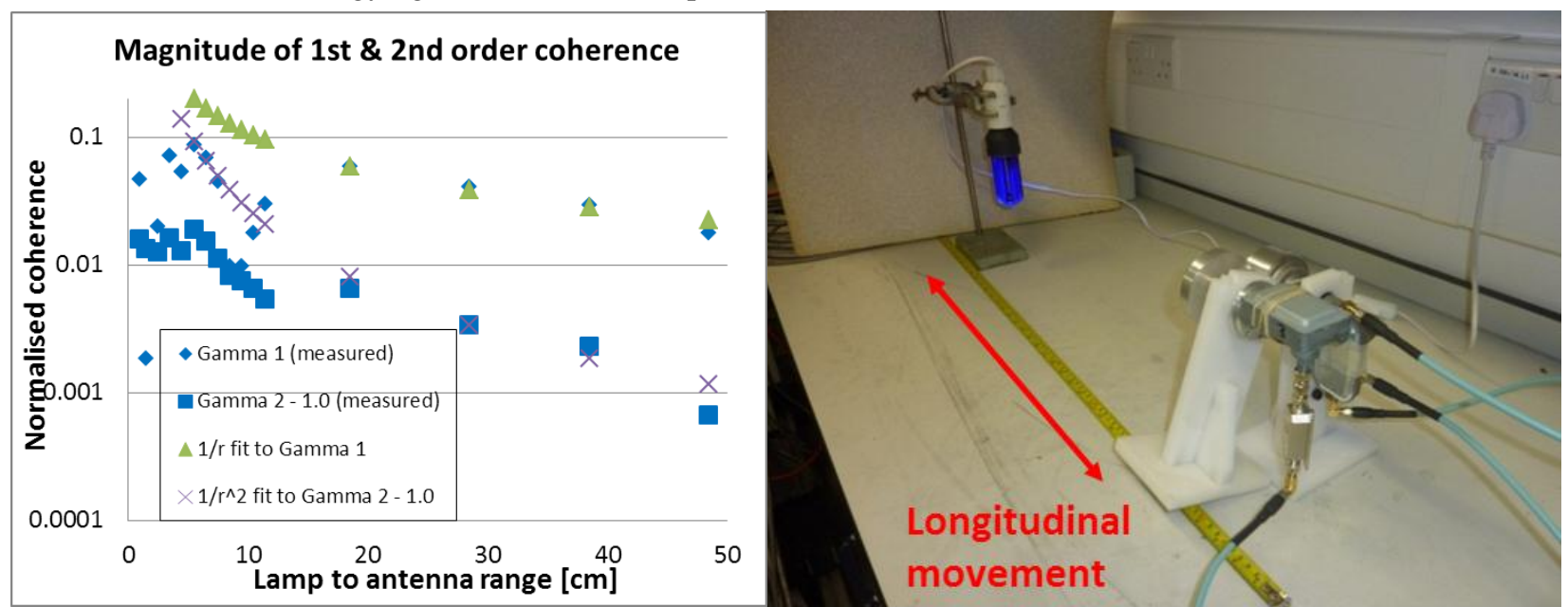

Figure 9: Variations in the magnitude of the coherence functions, $\gamma^{(1)}$ and $\left(\gamma^{(2)}-1\right)$ as a function of range are detected by making measurements of a low energy discharge lamp as it is moved longitudinally outwards from the LNB feeds to a range of $50 \mathrm{~cm}$. A $1 / R$ and $1 / R^{2}$ fit to the data is shown

Measurements of the variations in the coherence functions $\gamma^{(1)}$ and $\left(\gamma^{(2)}-1\right)$ were also made as the LNB feeds were varied in the polar direction at a fixed distance around the CFL bulb. These measurements were made to examine the expected reduction in coherence with increased subtended angle, as predicted by the van Cittert Zernike theorem. This may be important for portal security screening when threats are viewed using a near-field correlation beam-forming system with receivers viewing from widely dispersed angles. The expected effect can be thought of as a spatial decorrelation (fringe washing) phenomenon. Respective values of 0.06 and 0.01 were measured for $\gamma^{(1)}$ and $\left(\gamma^{(2)}-1\right)$, which is well above the noise level.

The above system could be further developed into a system to study the generation of entangled photons for quantum communications. Using two polarimetric receivers in each arm of the interferometer the Bell's inequality ${ }^{[9]}$ could be 
evaluated in LabVIEW to confirm the existence of the entangled photons. However, for this to happen it is likely that the existing system would need to be improved in its precision and stability.

\section{CONCLUSIONS}

Satellite LNBs have been investigated for use in radiometry and have been measured to have noise temperatures ranging from $170 \mathrm{~K}$ to $300 \mathrm{~K}$. A power spectrum of the output power confirms the system as sensitive over the band $10.5 \mathrm{GHz}$ to $11.9 \mathrm{GHz}$ when an external $10 \mathrm{GHz}$ local oscillator was used. Using LNBs and additional amplifiers, filters, mixers and ADCs a combined amplitude and intensity interferometer was developed and operated. Using this system first and second order coherence functions were measured from a CFL bulb and found to agree with expected results. The total cost of components in the system was around $£ 15 \mathrm{~K}$, and as such provides a convenient test bed for testing interferometric algorithms and doing basic interferometric experiments.

\section{FUTURE WORK}

The simple laboratory set up of this LNB based interferometer could be extended by calibrating the voltages measured by the ADCs to get a more accurate measure of the coherence functions. The number of LNBs and other components could be increased to develop an aperture synthesis security screening portal demonstrator having tens or hundreds of channels. Further work could be done on understanding the magnitude of the first order coherence functions and fringe washing associated with antennas space far apart in the field, thus verifying predictions of the van Cittert Zernike theorem relevant for portal type imaging systems. In the area of intensity interferometry, experiments could be developed to recover phase using a triple product system and Cauchy-Riemann techniques. The system might be extended and improved in its precision and stability to enabled investigation into entangled millimetre wave photons and schemes for quantum communications.

\section{ACKNOWLEDGEMENTS}

The authors are greatly indebted to Professor Richard Winpenny and Med Benyezzar for providing laboratory space, the loan of electronic test gear and helpful advice in the development of digital systems. Shahram Amiri is to be commended on his skills in the adaption of satellite LNBs to take external oscillators. All are grateful to Professor Colin Bailey for providing funding for hardware under the Manchester EPS Dean's funding scheme.

\section{REFERENCES}

[1] Salmon, N.A. et al, "Interferometric aperture synthesis for next generation passive millimetre wave imagers", SPIE Europe Security+Defence, Millimetre Wave and Terahertz Sensors and Technology, Edinburgh, September, (2012)

[2] Salmon, N.A. "Minimising the costs of next-generation aperture synthesis passive millimetre wave imagers", SPIE Europe Security and Defence, 'Millimetre Wave and Terahertz Sensors and Technology', Prague, September, (2011)

[3] Breeds, J., 'The Satellite Book - A complete guide to satellite theory and practice', Swift Television Publications, (1997)

[4] Lyons, R.G., "Understanding digital signal processing, $3^{\text {rd }}$ Edition, Prentice-Hall, (2011)

[5] Hanbury-Brown, R, 'The intensity interferometer', Halsted Press, (1974)

[6] Thomson, A., Moran, M., Swenson, G, "Interferometry and Synthesis in Radio Astronomy”, Wiley, (2004)

[7] Loudon, R. 'The quantum theory of light', Clarendon, (1979)

[8] Born, M, and Wolf, E., "Principle of optics", Cambridge University Press, $7^{\text {th }}$ Edition, (2003)

[9] Mandel, L, Wolf, E. "Optical coherence and quantum optics", Cambridge University Press, (2008)

[10]Lohmann, A.W. \& Wirnitzer, B, “Triple Correlations”, Proc. IEEE, Vol. 72, No. 7, July, (1984)

[11] Holmes, R., \& Belen'kii, M.S., "Investigation of the Cauchy-Riemann equations for one-dimensional image recovery in intensity interferometry", J. Opt.Soc. Am. A, Vol. 21, No. 5, May, (2004)

[12] LeBohec, S, Holder, J., "Optical Intensity Interferometry with Atmospheric Cherenkov Telescope Arrays", Astrophysical Journal 649:399-405, (2006) 